\title{
CLASSIFIERS AND DECISION MAKERS
}

\author{
J. MONTERO \\ Faculty of Mathematics \\ Complutense University \\ Madrid, Spain \\ E-mail: javier_montero@mat.ucm.es
}

\begin{abstract}
The main objective of this paper is to point out the key relevance of the classification issue in mathematical modeling. In particular, it is stressed that standard logical structures are quite simple classification structures, where the allowed degrees of truth or falsehood are connected according to a linear ordering. But when viewed as a classification problem, much more complex logical structures appear in a natural way, showing in fact that the standard linear assumption was kind of artificial. Moreover, once we focus our attention on the classification problem we realize that a relevant portion of classical research devotes to decision making, therefore imposing some misleading restrictions: the true scientific issue is much more related to classification rather than to decision making.
\end{abstract}

\section{Introduction}

Mathematics model reality, but this modeling process is usually made from a particular philosophical position, which some times is not fully formalized but granted, and it explains why certain tools are chosen or rejected. Two centuries ago, for example, scientists use to search for an exact prediction of system behavior assuming a complete knowledge of its circumstances, so there was no room for any kind of uncertainty. If we are modeling human behavior, for example, and we postulate that we should not care about opinions but about acts, then we may be forced to model decision making procedures assuring consistent outputs, with no room for fuzziness (acts al always crisp, see Montero ${ }^{18}$ for a discussion on this issue). Moreover, a previous formal definition for consistency is then required (it has been traditionally associated to transitivity, but Bayesian Theory ${ }^{25}$ imposes completeness, in addition).

Within Fuzzy Sets Theory, an analogous decision making approach has produced a number of fuzzy transitivity definitions, always generalizing classical crisp transitivity ${ }^{22}$, sometimes with discouraging results ${ }^{21}$. Alternative 
approaches to fuzzy rationality ${ }^{12,13}$ are also focused from a decision making point of view, transitivity playing a key role.

In this paper it is pointed out that perhaps consistency of decision making should not be our objective as scientists, but consistency of the previous analysis. In fact, real decision makers do not hire us in order to make the decision instead of them, but in order to help them to get a better understanding of the problem they are faced to (see, e.g., Pearman et al. ${ }^{19}$ ). Mathematical models should always be viewed as decision aid tools (see Roy $\left.^{24}\right)$.

\section{Crisp consistency in decision making}

An nice example in Mathematics is the Bayesian Theory of Probability, where decision making is a corner stone.

As already pointed out in Montero-Mendel ${ }^{18}$, Bayesian Theory is built upon a crisp space of actions. In fact, in the Bayesian Theory "probability" does not exist, neither in the physical world or our mind, but "in the records of the gambles" we have agreed to accept in the past. Bayesians do not care if concepts are crisp or fuzzy. They are only sure about their own acts, no matter their own supporting arguments. Crispness assumption in the Bayesian model applies to acts, and the Bayesian model refers just to acts. It can be argued that although acts are always crisp, such a crispness does not apply to most decisions human beings take $\mathrm{e}^{27,24}$. Bayesian researchers will still claim that only occurrence of those crisp acts can be checked (the set of acts must be therefore well defined). Science can be built only from these observable facts, so every conceptual tool is operational ${ }^{18}$.

Bayesian Theory builds up a model for Probability on the basis of the decisions we make. Then, it can be proven that my acts can be explained by means of Probability, provided that these acts are consistent. Another key assumption is then the definition of consistency. In particular, it is being assumed that alternatives can be represented in the real line, therefore defining a linear order: we can always choose the best alternative among any pair, in such a way that transitivity holds.

A relevant part of applied mathematics is focused towards decision making. It is being assumed that the key issue is to analyze decision making, how to assure consistency of our acts. In this paper we shall discuss the role of decision making in mathematical modeling, pointing out that perhaps we should focus our attention in knowledge acquisition rather than decision making. Some interesting consequences will be then suggested. 
The concept of consistency or rationality may be not the same in those two different contexts, for example. Some assumptions may be natural within a classification problem, but forced in a decision making framework.

Crisp consistency use to be associated to transitivity and completeness, in such a way that decision makers are rational if and only if any set of alternatives they are shown, their preferences define a linear order. This approach can be justified in terms of decision making: whenever alternatives are linearly ordered, the best alternative is always defined for any subset of alternatives we may consider. But human beings violate quite often the linear order assumption: some times alternatives can not be compared, or transitivity does not hold. So, weaker conditions for transitivity have been proposed in the past. For example, indifference is obviously non transitive in practice (see, e.g., Montero ${ }^{12}$ ). Or perhaps we can just assure the existence of a small enough non-empty choice set, so a detailed analysis can be restricted only to few alternatives ${ }^{23}$.

\section{Fuzzy consistency in decision making}

Analogous decision making approach can be found in many classical papers devoted to fuzzy preference representation ${ }^{22}$. But if transitivity is frequently violated within a crisp context, standard fuzzy transitivities are even difficult to be checked. Therefore, instead of asking decision makers to revise their intensity values, we can try to search for a close enough consistent fuzzy preference relation, expecting it will be accepted after all ${ }^{20}$. But these acceptance may be not trustable, since most decision makers are far away from a direct intuition of big data structures.

An interesting alternative, still under the same decision making framework, has been proposed in the past by Montero ${ }^{12,13,16}$ and CutelloMontero $^{4,5,6}$, by considering consistency as a fuzzy property. This approach allowed, see next section, an interesting alternative result to Arrow's Paradox, showing that this classical results can be viewed as the impossibility of a perfect aggregation rule ${ }^{12}$. Similar fuzzy approaches have been tried by other authors, still modeling decision making fuzzy consistency.

\section{Consistency in group decision making}

Let us consider, for example, group decision making problems: how to obtain an amalgamated preference for a finite family of (at least two) individuals expressing their preferences about a finite number of alternatives.

In case those preferences are assumed to be crisp, Arrows theorem ${ }^{2}$ 
shows that in case of dealing with at least three alternatives, there is no amalgamating democratic rational rule (consistent and ethical). Since then, quite a number of alternative impossibility theorems have been obtained ${ }^{9,26}$, introducing different formal definitions for consistent and ethical. As pointed out in Montero $^{12}$, a key unexplained assumption within this context is the crisp view of these two properties (either the group is consistent or not, either it is ethical or not), and the crisp support for their preferences (either one alternative is preferred to another alternative or it is the other way round). Here we find that consistency for group and individuals is conceived from a decision making point of view (a linear order is being imposed for groups and individual preferences).

Within a fuzzy context we may escape to Arrow's impossibility theorem, in the sense that we can measure degrees of verification of being consistent and ethical, no matter their crisp definition ${ }^{12,13}$. Hence, instead of searching for a perfect rule, we can search for some kind of compromising rule, consistent enough and ethical enough (see also Montero ${ }^{11,14}$ ). Still, this result was still conceived from a decision making viewpoint (it was basically measured the degree consistency of the associated decision making problem). Although alternative consistency measures can be considered ${ }^{5}$, most of existing consistency measures ${ }^{16,4,6}$ take mainly into account the associated decision making problem.

Nevertheless, Black ${ }^{3}$ proved a true possibility theorem within the crisp context, but introducing a key restriction on individual preferences, so they are single peacked, i.e., all those preferences can be represented within a real line in such a way that between every pair of alternatives each indivudual always prefer the closest one to the best one, which of course exists. But it should be realized that this restriction seems too sophisticated... unless such a particular structure is a priori imposed. This has been the situation in many democratic countries, where people were told to choose among "extreme left", "moderate left", "moderate right" and "extreme right" parties. From a decision making point of view, Black's single peakedness condition sounds strange, but such a property may be considered natural within a classification problem, were the classes structure ${ }^{1}$ is given to us as data (see Montero ${ }^{15}$ for a fuzzy version of Blak's result).

\section{Final comments}

As pointed out above, conceiving decision making as a goal may be misleading our research, bringing us to a hostile framework were no solution 
can be obtained. Good decision makers need not to be consistent, since the problems they face are always different (in place, time or any other circumstance). We can only stress consistency of the previous analytic model, which may produce certain classification, of course subject to uncertainty. For example, consistency within classification ${ }^{1,17}$, may be easier to assume than consistency within decision making. Hence, we should focus our studies in developing representation tools ${ }^{7,8}$ for decision aiding rather than proper decision making tools ${ }^{10,24,27}$. Weaker consistency conditions will appear, just pursuing an illustrative representation of decision maker preferences. In this sense, it is claimed much more theoretical effort in developing consistent fuzzy representation tools.

\section{Acknowledgments}

This Research has been partially supported by the Government of Spain, grant BFM2002-0281.

\section{References}

1. A. Amo, J. Montero, G. Biging and V. Cutello: Fuzzy classification systems. European Journal of Operational Research 156:495-507 (2004).

2. K.J. Arrow: Social Choice and Individual Values. Wiley, New York (1951, 1964).

3. D. Black: The Theory of Committees and Elections. Cambdrige University Press, Cambridge, 1958.

4. V. Cutello and J. Montero: A characterization of rational amalgamation operations. Internatioanl Journal of Approximate reasoning 8, 325-344 (1993).

5. V. Cutello and J. Montero: Fuzzy rationality measures. Fuzzy Sets and Systems 62, 39-44 (1994).

6. V. Cutello and J. Montero: Equivalence and compositions of fuzzy rationality measures. Fuzzy Sets and Systems 85, 31-43 (1997).

7. J. González-Pachón, D. Gómez, J. Montero and J. Yáñez: Soft dimension theory. Fuzzy Sets and Systems 137, 137-149 (2003).

8. D. Gómez, J. Montero, J. Yáñez, J. González-Pachón and V. Cutello: Crisp dimension theory and valued preference relations. International Journal of General Systems 3:115-131 (2004).

9. J.S. Kelly: Arrow Impossibility Theorems. Academic Press, New York, 1978.

10. C. Macharis and J.P. Brans: The GDSS Promethee Procedure. Journal of Decision Systems 7, 283-307 (1998).

11. J. Montero: A note on Fung-Fu's theorem. Fuzzy Sets and Systems 13, 259$269(1985)$

12. J. Montero: Arrow's theorem under fuzzy rationality. Behavioral Science 32, 267-273 (1987). 
13. J. Montero: Social welfare functions in a fuzzy environment. Kybernetes 16, 241-245 (1987).

14. J. Montero: Aggregation of fuzzy opinions in a non-homogeneous group. Fuzzy Sets and Systems 25, 15-20 (1988)

15. J. Montero: Single-peakedness in weighted aggregation of fuzzy opinions in a fuzzy group. Theory and Decision Library (B) 18, 163-171 (1990).

16. J. Montero: Rational aggregation rules. Fuzzy Sets and Systems 62, 267-276 (1994).

17. J. Montero and D. Gómez: Preferences, classification and intuitionistic fuzzy sets. Proceedings EUSFLAT'03, University of Zittau, 2003; pp. 187-192.

18. J. Montero and M. Mendel: Crisp Acts, Fuzzy Decisions; in S. Barro, A. Sobrino and A. Bugarin, eds.: Advances in Fuzzy Logic. Universidad de Santiago, Santiago de Compostela, 1998; pp. 219-238.

19. J. Montero, A. Pearman and J. Tejada: Fuzzy multicriteria decision support for budget allocation in the transport sector. Top 3, 47-68 (1995).

20. J. Montero and J. Tejada: Some problems on the definition of fuzzy preference relation. Fuzzy Sets and Systems 20, 45-53, (1986).

21. J. Montero and J. Tejada: A necessary and sufficient condition for the existence of Orlovsky's choice set. Fuzzy Sets and Systems 26, 121-125 (1988).

22. S.A. Orlovski: Calculus of Decomposable Properties, Fuzzy Sets and Decisions. Allerton Press, New York, 1994.

23. P.K. Pattanaik: Voting and Collective Choice. Cambdrige University Press, Cambridge, 1971.

24. B. Roy: Decision science or decision-aid science. European Journal of Operational Research 66 (1993), 184-203.

25. L. Savage: The Foundations of Statistics. Wiley, New York, 1954.

26. A.K. Sen: Collective Choice and Social Welfare. Holden-Day, San Francisco, 1970.

27. G. Shafer: Savage revisited (with discussion). Statistical Science 1 (1986), 463-501. 